\title{
Tachistoscope simulation package
}

\author{
STEVEN P. SHWARTZ \\ Johns Hopkins University, Baltimore, Maryland 21218
}

\begin{abstract}
A system of FORTRAN-callable subroutines is described that enable a PDP-11/20 computer to function as a multichannel tachistoscope. Features of the system include multiple display buffering, dynamic buffer allocation, control of character size and density, provisions for user-generated characters and symbols, and routines for recording of both vocal and buttonpress responses.
\end{abstract}

The tachistoscope simulation package (TSP) is a system of FORTRAN-callable subroutines, written in the MACRO-11 assembler language, that allows the Digital Equipment Corporation (DEC) PDP-11/20 computer to function as a multichannel tachistoscope. In order to use the TSP system, the user must write a program in FORTRAN that calls subroutines from the package that control stimulus generation and display, timing, and recording of responses. The TSP system allows the experienced programmer to take advantage of the full range of flexibility of the FORTRAN programming language.

A running program is created by entering the FORTRAN code onto disk using a text editor, after which it is compiled and linked (and debugged). Counterbalancing, randomization, and stimulus generation can be performed on-line (e.g., during the intertrial intervals), or in advance of the session, with the required information being stored in an input file and read in during program execution. The use of input files to the FORTRAN program is not necessary, but can be useful, particularly when it is desirable to run the same program on several sets of stimuli or when on-line counterbalancing, randomization, or stimulus generation are not desirable (e.g., counterbalancing might be required across subjects). If input files are to be used, they can be created by a separate program, or can be entered using a text editor. The structure of such input files is defined by the user-written FORTRAN program and its format can therefore be one that is convenient for the experimenter. On the output side, the storage of experimental data is flexible and efficient. Condition codes and reaction times (RTs) can be written onto storage media (e.g., disk or line printer) in a way that minimizes storage space and maximizes ease of access for data analysis either by computer or by hand.

The TSP routines require the following DEC hardware (or their current equivalents): A VR14 pointplot display scope, a KW11P real-time clock, and a DR11 parallel interface (interrupt register). A built-in

The author would like to acknowledge the many helpful suggestions of Bert F. Green, Thomas G. Land, and Joseph Griffin. Randi Martin contributed the VRLIN subroutine. character set (all printing ASCII characters, except lowercase letters) is stored by the TSP system in memory and the system provides a facility whereby the user can generate his own characters for display. Further features include multiple display buffers, dynamic buffer allocation, control of character size, and facility for recording RTs. Table 1 contains a list of the TSP subroutines.

\section{INITIALIZATION AND BUFFER ALLOCATION}

The system is activated by a call to an initialization subroutine, BEGIN, which loads the interrupt vectors

Table 1

Summary of TSP Subroutines

\begin{tabular}{|c|c|}
\hline \multicolumn{2}{|r|}{ Stimulus Generation } \\
\hline LDBUF & -loads a display buffer. \\
\hline SQUASH & -zeroes one or more display buffers. \\
\hline COPY & $\begin{array}{l}\text {-appends contents of one or móre source } \\
\text { buffers to a target buffer. }\end{array}$ \\
\hline RNDBUF & $\begin{array}{l}\text {-randomizes order of dots in one or more } \\
\text { display buffers. }\end{array}$ \\
\hline VRLIN & -draws line between two points. \\
\hline NEWLET & $\begin{array}{l}\text {-replaces built-in character with user-defined } \\
\text { character. }\end{array}$ \\
\hline LSIZ & $\begin{array}{l}\text {-specifies letter size and intercharacter } \\
\text { spacing. }\end{array}$ \\
\hline \multicolumn{2}{|r|}{ Display Control } \\
\hline TRNON & $\begin{array}{l}\text {-enables scope interrupt; displays contents of } \\
\text { specified buffer. }\end{array}$ \\
\hline TRNOF & $\begin{array}{l}\text {-disables scope interrupt; clears display } \\
\text { screen. }\end{array}$ \\
\hline \multicolumn{2}{|r|}{ Timing and Response Collection } \\
\hline WATFOR & \multirow{6}{*}{$\begin{array}{l}\text { - waits for the specified time. } \\
\text {-zeroes clock. } \\
\text {-waits until the specified time. } \\
\text { - returns current time on clock. } \\
\text { - waits for the specified time or a response. } \\
\text { - returns number of responses on each of two } \\
\text { buttons since last call. }\end{array}$} \\
\hline ZERO & \\
\hline WATIL & \\
\hline KLOK & \\
\hline WRESP & \\
\hline ANTIC & \\
\hline VKSET & \multirow{4}{*}{$\begin{array}{l}\text {-sets cutoff value for voice-key response. } \\
\text {-zero voice-key timer. } \\
\text {-checks for vocal response. } \\
\text { - returns time on voice-key clock. }\end{array}$} \\
\hline FLGSET & \\
\hline IVKCK & \\
\hline VKKLOK & \\
\hline \multicolumn{2}{|r|}{ Miscellaneous } \\
\hline BEGIN & \multirow{3}{*}{$\begin{array}{l}\text {-initializations; buffer allocation. } \\
\text {-program termination. } \\
\text {-sets software device interrupt priorities. }\end{array}$} \\
\hline END & \\
\hline PRI & \\
\hline
\end{tabular}


for the CRT display screen, real-time clock, and response key registers. BEGIN takes as arguments the first and last elements of each of up to 50 arrays that the system subsequently uses as its display buffers. For example,

\section{CALL BEGIN (BUF1(1),BUF1(2000),BUF2(1),BUF2(500))}

will allocate two display buffers 4,000 and 1,000 words in length, respectively (the FORTRAN compiler allocates 2 words of storage for each real variable). Thus, both the number of buffers to be used and the amount of space reserved for each buffer is specific to the application program. This algorithm allows the user to minimize the amount of core required for display storage.

The purpose of multiple buffers is to enable construction of all stimulus displays before the trial begins, enabling virtually instantaneous transition from one display to the next. The utility of multiple buffers can be seen in the program for the Sperling, Budiansky, Spivak, and Johnson (1971) visual search task (see Figure 2) described later in this paper. This experiment requires successive presentation of 26 stimulus arrays. Using multiple buffers, the interstimulus interval (ISI) is constant and short because all displays are computed before the trial starts. If a system had only two buffers, one display would have to be constructed while the other display was being presented. The time it takes to create a display is proportional to the display size, thereby putting a bound on the minimum ISI that increases with the display size.

\section{CONSTRUCTION OF STIMULI}

All stimuli are constructed in a 7 by 5 matrix of dots (or several 7 by 5 matrices), in which each dot is either on or off (see Gilman \& Buckley, 1973, for a discussion of this method of stimulus construction). The system maintains in memory the bit patterns of all printing ASCII characters, except lowercase letters. In addition, any of these standard characters can be replaced with a 7 by 5 matrix, constructed by the user, by calling the subroutine NEWLET, which takes as arguments the character to be replaced and the desired new bit pattern. Stimulus configurations that require dot matrices with a finer grid can be constructed by positioning several 7 by 5 matrices adjacently. Thus, stimuli can be constructed in dot matrices of arbitrary density, limited only by the absolute grid density of the display scope (a 4,095 by 4,095 grid in the case of the VR14) and by memory considerations (each plotted point requires two words of core storage). ${ }^{1}$ The size of the stimulus display can be adjusted before loading the display buffer by calling the subroutine LSIZ, which takes as one of its arguments the number of scope units to be put between dots in the 7 by 5 matrices.

Stimuli can be loaded into each of the allocated display buffers independently. When loaded, a buffer contains a list of $X, Y$ coordinates of points that define the stimulus display.

Each buffer can be loaded by a call to the subroutine LDBUF, which takes as arguments the $X, Y$ coordinates of the first character to be displayed, the buffer number, a desired angle of orientation in degrees, and the ASCII code of each character to be displayed. Spacing between characters can be changed by a call to LSIZ, which takes as arguments the interdot spacing (see above) and the intercharacter spacing in scope units. Alternatively, intercharacter spacing can be controlled by successive calls to the LDBUF routine. For example,

\section{CALL LDBUF(-2000,2000,1,180,'H, 'E', 'L', 'L','O')}

will load Buffer 1 with the coordinates of the points that will serve to display the word "HELLO" inverted (180-deg rotation) in the upper left corner of the screen.

\section{DISPLAY CONTROL}

The contents of a display buffer will appear on the display screen when the scope interrupt is enabled via the subroutine call CALL TRNON(5), which displays the contents of the specified display buffer (in this case, Buffer 5) on the display screen. Following the call to TRNON, the stimulus in the display buffer will remain on the display screen until CALL TRNOF is issued, which disables the scope interrupt but leaves the contents of all display buffers intact. Composite displays can be created in a single buffer either by multiple LDBUFs, or by utilizing the subroutine COPY, which will append the contents of one display buffer to a second display buffer. The contents of any buffer can be zeroed by a call to subroutine SQUASH, which takes as arguments the numbers of the buffers to be zeroed. For example, CALL SQUASH $(2,3,5)$ empties Buffers 2, 3 , and 5 .

The scope interrupt service routine is transparent to the user, although it is turned on and off by the subroutines TRNON and TRNOF, respectively. The service routine brightens a single point each time an interrupt occurs, and on successive interrupts, it cycles sequentially through the specified display buffer. In order to avoid a detectable refresh pattern, a randomization subroutine, RNDBUF, can be called to randomize the order of the points in a display buffer. ${ }^{2}$

\section{TIMING AND RESPONSE COLLECTION}

Five timing routines are provided by the system. CALL WATFOR (ISEC,MSEC) zeroes the clock and causes the program to wait for the specified time (in seconds and milliseconds). CALL WRESP (ISEC,MSEC, KEY,IRT1,IRT2) waits until one of the two response keys has been pressed or until a specified number of seconds (ISEC) and milliseconds (MSEC) have elapsed. WRESP also zeroes the clock and returns the reaction 
time, in seconds and milliseconds (IRT1,IRT2), and number of the response key (KEY) pressed. Often, however, it is not desirable to reset the clock before the wait, but rather, to wait until a specified amount of time has elapsed since the last time the clock was zeroed. The subroutines ZERO and WATIL provide this facility. In the example below, the clock is set to zero by a call to subroutine ZERO, some intermediate computations and input-output (I/O) are performed that take an indeterminate amount of time, then WATIL is called to wait until the specified amount of time has elapsed since the call to ZERO.

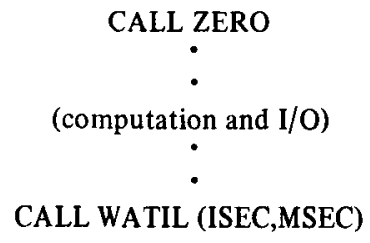

An additional timing routine, CALL KLOK (ISEC, MSEC), returns the elapsed time since the last call to ZERO. This routine is useful in cases in which it is desirable to wait until a certain amount of time has elapsed or until some event has occurred. The user program can do this by putting both a check for the clock and a check for the desired event in a loop.

\section{PROGRAM TERMINATION}

The subroutine END restores the monitor clock and must be called before program termination in order to insure proper system functioning.

\section{EXAMPLES}

\section{Memory Scanning Task}

Figure 1 shows the program for a simple Sternberg (1966) task: On each trial, a subject is presented with a digit; the subject's task is to press one response button if the number is a member of the positive set, and a second button if it is not.

\section{Visual Search Task}

The experimental paradigm of Figure 2 is the visual search task used by Sperling et al. (1971). The paradigm is one that greatly taxes the resources of a computer system and is virtually impossible to instrument without a computer. The subject's task is as follows: On each trial, the subject is presented with 6-12 nine-letter displays, followed by a critical display containing either nine letters or, with probablity $50 \%$, eight letters and the digit 5 , followed by 12 more nine-letter displays. Each display is presented very quickly $(40-\mathrm{msec}$

Figure 1. Sample program for Sternberg (1966) memory scanning task.

DIMENSION BUF (50)

CALL BEGIN (BUF (1), BUF(50))

DO 200 NTRIAL $=1,100$

$N=9 * \operatorname{RAN}(\mathrm{I} 1, \mathrm{I} 2)+1$

CALL LDBUF $(0,0,1,0, \mathrm{~N}+48)$

CALL TRNON (1)

CALL WRESP (ISEC, MSEC, KEY, 10,0)

CALL SQUASH ( 1 )

$\mathrm{RT}=\mathrm{ISEC}+\mathrm{MSEC} / 1000$. WRITE $(1,100) \mathrm{N}, \mathrm{KEY}, \mathrm{RT}$

100 FORMAT (2I 1, F5.3)
;Perform initializations/

; one buffer only ;100 trials

;Pick a number from 1 to 9

; to be stimulus

; Load number into display

; buffer

iDisplay contents of

- buffer 1

;Wait for response or for

i 10 seconds

;Clear screen / buffer 1

;Compute RT

;Output trial results

;1. 5 second rest 
Figure 2. Sample program for Sperling et al. (1971) visual search task.

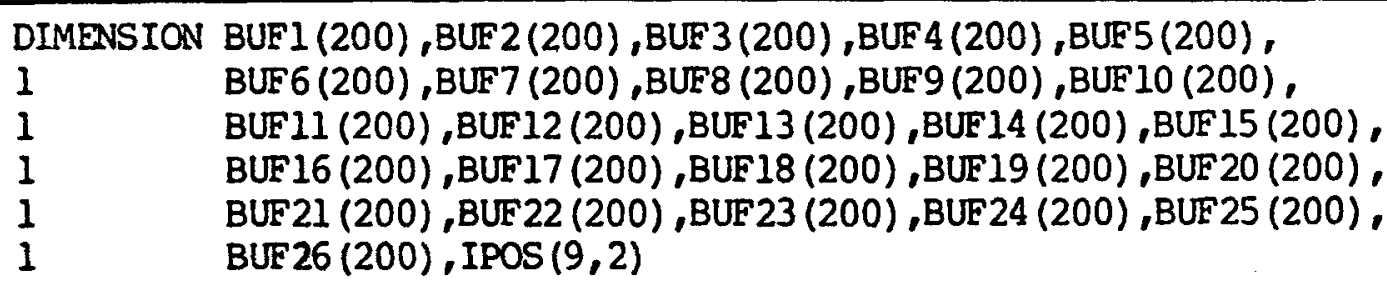

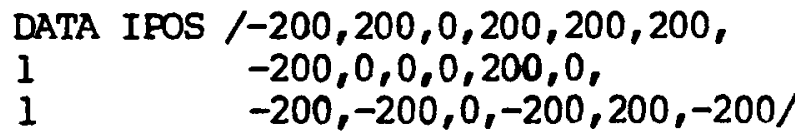

; x,y coord.s for

; 9-letter displays

CALL BEGIN (BUFI (1),BUFI (200), initializations/

1 BUF2(1),BUF2 (200),BUF3(1),BUF3(200), ; buffer allocation

1 BUF4(1),BUF4(200),BUF5(1),BUF5(200), ; for 26 buffers

1 BUF6 (1), BUF6 (200), BUF7 (1),BUF7 (200),

1 BUF8 (1) ,BUF8 (200), BUF9 (1) ,BUF9 (200),

1 BUF 10 (1) ,BUF 10 (200), BUF 11 (1) ,BUF 11 (200),

1 BUF12 (1),BUF12 (200), BUF13 (1) ,BUF13 (200),

1 BUF14 (1) ,BUF14 (200), BUF15 (1),BUF15 (200),

1 BUF16 (1),BUF16 (200),BUF17(1) ,BUF17 (200),

1 BUF18 (1) ,BUF18 (200), BUF19 (1), BUF19 (200),

1 BUF20 (1), BUF20 (200), BUF2l (1), BUF2l (200),

1 BUF22 (1), BUF22 (200), BUF23 (1), BUF23 (200),

1 BUF24 (1) ,BUF24 (200), BUF25 (1) ,BUF25 (200),

1 BUF26 (1) ,BUF26 (200))

C Create warning display in buffer 1 .

CALL NEWLET(' ',0,0,"10,0,0) ;Change period character ; to centered dot

DO $50 \quad I=1,9$

- For each of 9 locations

CALC LDBUF (IPOS $\left.(I, 1), \operatorname{IPOS}(I, 2), 1,0,0^{\prime}\right)$

50 CONTINUE

; Load a dot

DO 500 NTRIAL $=1,50$

$\mathrm{NBEF}=7 \star_{\mathrm{RAN}}(\mathrm{I} 1, \mathrm{I} 2)+6$

NCRIT=NBEF +1

NTOT $=$ NCRIT +12

$\mathrm{NCDT}=2 * \operatorname{RAN}(I 1, I 2)+1$
; 50 trials

;6-12 initial stimuli

-Critical display

; Total number of displays

-NCDT=1 Critical Display Trial

;NCDT=2 Non-critical Display

Trial

C Create non-critical displays.

DO $100 \quad I=1$, NTOT

CALL SQUASH (I)

IF (I.EQ.NCRIT.AND.NCDT.EQ.1) GOTO 100 ;Skip if critical display

DO $100 \mathrm{~J}=1,9$

$L E T=64+26 \star R A N(I 1, I 2)$
;For each of the NTOT stimuli ; Empty display buffer I

; For each of 9 display locations ;Pick a letter 
Figure 2 Continued
CA.LL LDBUF (IPOS $(J, 1), \operatorname{IPOS}(J, 2), I, 0, \operatorname{LET})$
100 CONTINUE
iLoad letter into buffer I
C Create critical displays.
IF (NCDT.EQ.2) GOTO 300
;Critical display needed?
$L O C=9 * \operatorname{RAN}(I 1, I 2)+1$
DO $200 \quad I=1,9$
IF (I.EQ. LOC) GOTO 200
LET $=64+26 \star R A N(I 1, I 2)$
iPick critical display location
; For 9 display locations
CALL LDBUF (IPOS $(I, 1)$, IPOS (I, 2$),$ NCRIT , O, LET)
;Skip if critical location
iPick a letter
200 CONTINUE
; Load letter into buffer I

CALL LDBUF (IPOS (LOC, 1), IPOS (LOC, 2 ),NCRIT, 0, '5')
;Load critical stimulus into
; buffer NCRIT

C Start trial.

300

CALL WATIL $(5,0)$

i5 second ISI

DO $400 \quad I=1$, NTOT

;NTOT stimulus displays

CALL TRNON(I)

CALL WATFOR $(0,40)$

CALL TRNOF

400 CONTINUE

;Present buffer I to subject

iWait 40 milliseconds

;Turn off display

CALL ZERO

; Set clock to zero to time ISI

500 CONTINUE

CALL END

iRestore monitor clock

END

exposure duration) and the subject's task is to decide if a 5 was presented in any of the displays. The subject responds after all the displays have been presented, and accuracy is the dependent variable.

The aspect of this task that is difficult for the computer system is the requirement that up to 26 different stimulus displays must be presented in rapid sequence. The program in Figure 2 handles this problem by utilizing the multiple buffers that are available in the TSP system. All 26 stimulus displays are constructed before the trial begins, and the program simply tells the scope interrupt service routine which buffer to display (using the TRNON subroutine). This algorithm allows for a very fast ISI and one that is essentially constant for all display sizes. The disadvantage of this algorithm is that the amount of memory required to run the program increases as both display size and the maximum number of displays increase, resulting in an upper bound on the product of display size and the number of displays and/or trials. An alternative approach would be to sacrifice efficient interstimulus timing for efficient use of memory, by loading one buffer while another buffer is being displayed. This algorithm requires only two buffers, and the amount of memory required is independent of the number of stimuli and/or trials. The disadvantage of this algorithm, however, is that the time required to load a buffer places a minimum bound on the ISI that is dependent on the maximum display size.

\section{REFERENCES}

Gilman, C. B., \& Buckley. P. Software character generation. Behavior Research Methods \& Instrumentation, 1973. 5, 228-230. 
SPerling, G. Flicker in computer-generated displays: Selecting a CRO phospor and other problems. Behavior Research Methods \& Instrumentation, 1971, 3, 151-157.

Sperling, G., Budiansky. J., Spivak, J. G., \& Johnson, M. C. Extremely rapid visual search: The maximum rate of scanning letters for the presence of a numeral, Science, 1971, 174. 307-311.

Sternberg, S. High-speed scanning in human memory. Science, 1960, 153, 652-654.

Taylor, G. A., Klitzke, D., \& Massaro, D. W. A visual display system for reading and visual perception research. Behavior Research Methods \& Instrumentation, 1978, 10, 148-153.

\section{NOTES}

1. Unfortunately, this algorithm does not minimize display flicker, a problem that results from having so many dots in the display that the dots decay before they can be refreshed, giving the display the appearance that it is flickering. For better algorithms with respect to the flicker problem, see Sperling (1971).

2. Of course, positioning several matrices next to each other requires a certain amount of time and programming effort. Taylor, Klitzke, and Massaro (1978) recently described an algorithm for constructing displays of arbitrary grid density that does not require pasting together several small submatrices. 